\title{
MÉTODOS DE DELIMITAÇÃO DOS LENHOS JUVENIL E ADULTO DE TRÊS FOLHOSAS E PROPRIEDADES BIOMÉTRICAS DE SUAS FIBRAS ${ }^{1}$
}

\author{
Rafael de Avila Delucis², Darci Alberto Gatto ${ }^{3}$, Diego Martins Stangerlin ${ }^{4}$ e Rafael Beltrame ${ }^{5}$ \\ RESUMO - Diante da importância do conhecimento das respectivas regiões do lenho juvenil e adulto para \\ a indústria de base madeireira, este estudo foi realizado com o objetivo de delimitar, por meio das metodologias \\ de retas de regressão e do incremento anual, as regiões dos lenhos juvenil e adulto das madeiras de açoita- \\ cavalo, nogueira-pecã e plátano. O parâmetro anatômico utilizado nas delimitações foi o comprimento de \\ fibra. Adicionalmente, foram medidas outras propriedades anatômicas: diâmetro de fibra, diâmetro de lume \\ e espessura de fibra. As propriedades das fibras foram descritas estatisticamente em razão da diferenciação \\ entre os lenhos proposta em cada metodologia, a fim de comparação. Conforme os métodos das retas de regressão \\ e do incremento anual, as idades de segregação entre os lenhos foram de 21 e 19 anos para a madeira de açoita- \\ cavalo e 16 e 14 anos para a madeira de nogueira-pecã. Para a madeira de plátano, ambas as metodologias \\ definiram que o lenho segregou com 14 anos. Pode-se verificar que não houve distinção entre os resultados \\ obtidos com as duas metodologias de delimitação dos lenhos juvenil e adulto, exceto quanto ao comprimento \\ de fibras do lenho juvenil. O método do incremento anual foi considerado preferencial, em razão da fonte \\ de erro verificada no método das retas de regressão.
}

Palavras-chave: Luehea divaricata; Carya illinoinensis; Platanus acerifolia.

\section{DELIMITATION METHODS OF JUVENILE AND MATURE WOODS OF THREE HARDWOODS AND BIOMETRIC PROPERTIES OF THEIR FIBERS}

\begin{abstract}
Before the importance of knowing the respective regions of juvenile and mature wood for the wood industry, this study was conducted with the objective of defining, through methodologies of regression lines and annual increment, the regions of juvenile and mature wood of açoita-cavalo, pecan and sycamore. The anatomical parameter used in the delimitations was fiber length. In addition, others anatomical properties were measured: fiber diameter, lumen diameter and fiber thickness. The fiber properties were statistically described according to the difference between woods proposed in each methodology, for comparison purposes. According to methods of regression lines and annual increment, the segregation ages between woods were 21 to 19 years for açoita-cavalo and 16 to 14 years for pecan. For sycamore wood, both methodologies defined that the wood segregated with 14 years. It may be verified that there was no difference between the results obtained with the two delimitation methodologies of juvenile and mature woods, except for fiber length of juvenile wood. The method of annual increment was considered preferred due to the error source verified in the regression lines.
\end{abstract}

Keywords: Luehea divaricata; Carya illinoinensis; Platanus acerifolia.

\footnotetext{
${ }^{1}$ Recebido em 26.09.2013 aceito para publicação em 07.08.2014.

${ }^{2}$ Programa de Pós-Graduação em Ciência e Engenharia de Materiais, Universidade Federal de Pelotas, UFPEL, Brasil. E-mail: <r.delucis@hotmail.com>.

${ }^{3}$ Centro de Desenvolvimento Tecnológico, Universidade Federal de Pelotas, Brasil. E-mail: <darcigatto@yahoo.com.br>. ${ }^{4}$ Instituto de Ciências Agrárias e Ambientais, Universidade Federal de Mato Grosso, UFMT, Brasil. E-mail: <stangerlin@cpd.ufmt.br>. ${ }^{5}$ Centro de Engenharias, Universidade Federal de Pelotas, Brasil. E-mail: <beltrame.rafael@yahoo.com>.
} 


\section{INTRODUÇÃO}

Tanto do ponto de vista anatômico quanto químico, a heterogeneidade é uma das principais características da madeira, sendo a causa de grandes prejuízos em procedimentos tecnológicos, abrangendo todas as etapas do processamento de produtos madeireiros, desde a colheita de árvores até o beneficiamento de tábuas. Como tema de estudos sobre a qualidade da madeira, ressaltam-se as comparações de propriedades tecnológicas na direção radial do tronco e, em especial, entre os lenhos juvenil e adulto, já que nesse contexto o comportamento desses parâmetros da madeira se distingue de variadas maneiras (NUGHORO et al., 2012; SAMUELSON et al., 2013; BOUSLIMI et al., 2014).

O lenho juvenil é a madeira formada desde os primeiros anos da vida do vegetal a partir de suas células meristemáticas localizadas no câmbio. Possui forma aproximadamente cilíndrica e se estende da base até o topo da árvore, estando contido inclusive em seus galhos. Com o desenvolvimento da árvore em altura, suas células de meristema apical vão progressivamente concentrando-se em posições axiais mais altas. Dessa maneira, o lenho adulto começa a ser formado à medida que diminui o efeito dos meristemas apicais na base do tronco da árvore (EVERT, 2006).

Na direção radial, o lenho juvenil caracteriza-se pelo aumento ostensivo no sentido medula-casca de propriedades anatômicas, como o comprimento e a espessura de fibras (LARA PALMA et al., 2010; GATTO et al., 2012); físicas como a massa específica (DELUCIS et al., 2013; PALERMO et al., 2013); e mecânicas, como os módulos de elasticidade e de ruptura (BAL et al., 2014; LOURENÇON et al.; 2014). À parte, alguns autores reportaram modificações químicas a partir da formação do lenho adulto em quantidade e constituição de alguns produtos extraíveis, como os compostos fenólicos e os terpenos (BAO et al., 2001; DUNISH et al., 2010).

Em comparação com o lenho adulto, genericamente o lenho juvenil apresenta: traqueídeos ou fibras mais curtos, lumes maiores, paredes celulares mais finas, células tangenciais menores, maior ângulo microfibrilar, menor porcentagem de lenho tardio, menor massa específica, maior contração longitudinal, menor resistência biológica, menor resistência e rigidez mecânica, maior formação de madeira de reação, textura mais grosseira e maior incidência grã-espiralada (BENDTSEN, 1978; ZOBEL; van BUIJTENEN, 1989; GATTO et al., 2012).
A transição entre as regiões contidas pelos lenhos juvenil e adulto ocorre de maneira gradual e, por esse motivo, sua delimitação é bastante dificultada (BENDTSEN, 1978; EVERT, 2006). Diversas propriedades anatômicas e físicas da madeira foram analisadas em estudos antecedentes, buscando verificar essas zonas: comprimento de fibra ou traqueídeo (SHIOKURA, 1982; LOO et al., 1985; LARA PALMA et al., 2010), ângulo microfibrilar, largura de anel de crescimento, densidade de anel de crescimento (ALTEYRAC et al., 2006) e massa específica (LOO et al., 1985; OLUWAFEMI, 2007).

Para Krahmer (1986), o comprimento de fibra é o melhor parâmetro para essa estimativa. De acordo com Nughoro et al. (2012), além do comprimento de fibra, outras propriedades anatômicas da madeira, como a espessura de parede, o diâmetro de lúmen e a frequência de vasos, estão intimamente relacionados com propriedades físicas, a exemplo da massa específica, e com propriedades mecânicas.

A proporção entre os lenhos juvenil e adulto pode variar entre árvores de uma mesma espécie e entre espécies, sendo condicionada a fatores como: área de plantio, herdabilidade genética, crescimento e produção hormonal, procedência da semente, condições atmosféricas e gestão silvicultural (BENDTSEN, 1978; LOO et al., 1985; ZHU et al., 2000; NUGHORO et al., 2012).

Com base na diferenciação das respectivas zonas dos lenhos juvenil e adulto, é possível definir técnicas de manejo florestal, visando suprimir a proporção de lenho juvenil. Dessa forma, é possível interferir positivamente em aspectos como a incidência de defeitos de secagem, propriedades físico-mecânicas da madeira, rendimento na polpação celulósica e qualidade na produção de papel (ALTEYRAC et al., 2006; NUGHORO et al., 2012; PALERMO et al., 2013). Segundo Alteyrac et al. (2005), em um plantio, um espaçamento menor entre árvores ocasiona menor taxa de crescimento oriunda do meristema cambial e, consequentemente, menores valores médios de largura de anéis de crescimento. Para esses autores, caso esse crescimento ocorra nos primeiros anos da vida dos vegetais, possivelmente será evidenciada menor proporção de lenho juvenil.

Assim, este estudo teve por objetivo delimitar os lenhos juvenil e adulto das madeiras de açoita-cavalo (Luehea divaricata Mart.), nogueira-pecã (Carya 
illinoinensis (Wangenh) K. Koch) e plátano (Platanus acerifolia (Ait.) Willd.), baseando-se no comprimento de fibras, por meio das metodologias de retas de regressão e do incremento anual, bem como comparar os resultados obtidos por meio dos dois métodos.

\section{MATERIAL E MÉTODOS}

O material de estudo foi selecionado de nove árvores adultas das espécies açoita-cavalo (Luehea divaricata), nogueira-pecã (Carya illinoinensis) e plátano (Platanus acerifolia), sendo três indivíduos de cada espécie com diâmetro à altura do peito (DAP; $1,3 \mathrm{~m}$ ) de no mínimo $30 \mathrm{~cm}$ e de boa forma florestal (fuste reto, tronco cilíndrico, ausência de bifurcação e bom estado fitossanitário). Adicionalmente, foram evitados indivíduos arbóreos de bordadura e localizados em terreno inclinado.

No caso das árvores de açoita-cavalo, a seleção ocorreu em florestas nativas, já as de nogueira-pecã e plátano foram selecionadas em florestas plantadas. Todas as florestas utilizadas localizavam-se na região fisiográfica conhecida como Encosta Superior Nordeste, próximo ao Município de Santa Maria, RS. Climaticamente, essa é uma região de transição entre os tipos Cfa e Cfb (clima temperado úmido e com chuvas bem distribuídas ao longo do ano) (EMBRAPA, 1999).

Após a derrubada, foi retirado de cada árvore um disco de $2 \mathrm{~cm}$ de espessura a $10 \mathrm{~cm}$ de altura da base, a partir do qual foi segmentada uma bagueta centrada pela medula e orientada em relação aos planos anatômicos, a qual foi imersa em uma solução de álcool 70\%. Após, selecionou-se o lenho inicial de cada anel de crescimento, o qual foi utilizado para maceração e produção de lâminas histológicas pelo método de Jeffrey (BURGER; RICHTER, 1991).

Foram avaliadas 30 fibras por anel de crescimento $\left(\mathrm{A}_{\text {cres }}\right)$, das quais foram medidos, com o auxílio de microscópio binocular, os parâmetros: comprimento de fibra $\left(\mathrm{L}_{\mathrm{fib}}\right)$, diâmetro de fibra $\left(\varnothing_{\text {fib }}\right)$ e diâmetro de lume $\left(\varnothing_{\text {lume }}\right)$. Por fim, foi calculada a espessura da parede celular $\left(\mathrm{e}_{\mathrm{fib}}\right)$, subtraindo-se os valores de $\varnothing_{\text {fib }}$ e $\varnothing_{\text {lume }}$ e dividindo o resultado por 2 para cada fibra.

Um detalhe pertinente de ser observado é que os anéis de crescimento das três madeiras analisadas apresentaram-se visíveis a olho nu, de maneira que a metodologia escolhida para dissociação das fibras e formação de lâminas histológicas dos elementos dissociados para avaliação de propriedades anatômicas dessas fibras foi perfeitamente passível de ser realizada. A contabilização dos anéis de crescimento determinou que as três árvores selecionadas de cada espécie tinham as idades de 51, 63 e 70 anos para açoita-cavalo, 30, 30 e 27 anos para nogueira-pecã e 24, 24 e 25 anos para o plátano. O que indica que para as árvores de nogueira-pecã e plátano houve diferenças entre os anos de plantio dentro de cada floresta. Todo o procedimento experimental de avaliação biométrica das fibras e identificação das espécies foi realizado no Herbário Florestal da Universidade Federal de Santa Maria (UFSM), em Santa Maria, RS.

Para determinação do ponto de início do lenho adulto a partir da medula, foram propostas duas metodologias, sendo em ambas utilizada como parâmetro a variação radial do $\mathrm{L}_{\text {fib }}$.

A primeira metodologia utilizada foi o procedimento que teve como precursor o trabalho de Loo et al. (1985). O método consiste em ajustar duas regressões lineares, uma para a parte ascendente da distribuição de pontos no gráfico ( $\mathrm{A}_{\text {cres }}$ versus $\mathrm{L}_{\text {fib }}$ ) e outra para a região em que a distribuição mostra tendência constante. Os limites dessas duas regiões são definidos visualmente no gráfico, e o ponto de início da formação do lenho adulto é determinado pela intersecção das duas retas geradas pelos modelos matemáticos. Para este trabalho, as retas foram obtidas por meio de análises de regressão simples, tendo como variáveis dependentes e independentes $\mathrm{L}_{\text {fib }}$ e $\mathrm{A}_{\text {cres }}$, respectivamente.

Como segundo método, foi utilizado um procedimento adaptado do proposto por Shiokura (1982). Neste trabalho, foi gerada uma equação logarítmica, na qual o $\mathrm{L}_{\text {fib }}$ foi descrito tendo como variável dependente o $A_{\text {cres }}$. Posteriormente, realizou-se o cálculo do incremento anual com base no valor médio do comprimento de fibra $\left(\mathrm{L}_{\text {fib }}\right)$ respectivo de cada anel de crescimento e a partir da medula. No anel de crescimento onde o incremento anual apresentou valor inferior ou igual a $1 \%$, passa a ser considerado o início do lenho adulto. A adaptação realizada para este estudo ocorreu no modelo matemático gerado, o qual, em vez de logarítmico, foi polinomial em razão da precisão de ajuste.

Para o delineamento experimental dos dados, empregou-se um plano inteiramente casualizado no esquema fatorial, tendo como fonte de variação a espécie em três níveis (açoita-cavalo, nogueira-pecã e plátano), 
o tipo de lenho em dois níveis (lenho juvenil e adulto) e o método de segregação dos lenhos em dois níveis (método das retas de regressão e do incremento anual). Com base na análise de variância multifatorial empregada, as variáveis foram descritas em função dos fatores que denotaram efeito significativo quando isolados e, ao mesmo tempo, interação entre si significativa. Em adição, foram realizados testes de comparação de médias Honestly Significantly Different (HSD) de Tukey a $1 \%$ de probabilidade de erro. Por fim, as propriedades anatômicas que apresentaram efeito significativo do fator "Método" foram descritas em razão do tipo de lenho atribuído à separação proposta por cada metodologia, comparado-as pela aplicação de análises de variância simples.

\section{RESULTADOS}

Na Tabela 1 são apresentados os modelos matemáticos para estimativa da segregação entre os lenhos juvenil e adulto, por meio das metodologias de retas de regressão e do incremento anual, os quais foram ajustados com nível de $1 \%$ de probabilidade de erro.

Com base no método das retas de regressão, as madeiras de açoita-cavalo, nogueira-pecã e plátano segregaram com 21, 16 e 14 anos de idade, respectivamente. Já com base no método do incremento anual, é possível afirmar que os lenhos das madeiras de açoita-cavalo e nogueira-pecã segregaram dois anos antes da idade determinada pelo método das retas de regressão, ou seja, aos 19 e 14 anos de idade, respectivamente. Entretanto, para a madeira de plátano, a idade de segregação não variou em razão dos dois métodos empregados para a segregação dos lenhos.

Os resultados obtidos por meio da análise de variância multifatorial empregada (ANOVA) dão conta de que para a propriedade $\mathrm{L}_{\text {fib }}$, os efeitos dos três fatores foram significativos (Espécie com $F=8998,18, p<0,01$; Tipo de Lenho com 3760,34, p<0,01; Método com $\mathrm{F}=9,78, \mathrm{p}<0,01)$. Para $\varnothing_{\text {fib }}$ e efib os fatores Espécie e Tipo de Lenho foram significativos ao nível de probabilidade de erro de $0,01 \%$ com valores de F de 6087,97 e 608,58, respectivamente. Já para o Ø $\varnothing_{\text {lume }}$, somente o fator Espécie foi significativo ( $F=6084,14 ; \mathrm{p}<0,01)$. Além disso, verificaram-se através da ANOVA interações significativas entre os fatores "Espécie" e "Lenho" para as propriedades: $\mathrm{L}_{\mathrm{fib}}(\mathrm{F}=259,45 ; \mathrm{p}<0,01), \varnothing_{\mathrm{fib}}$ $(F=32,38 ; p<0,01), \varnothing_{\text {lume }}(F=305,53 ; p<0,01)$ e $e_{\text {fib }}$ $(\mathrm{F}=569,54 ; \mathrm{p}<0,01)$.

$\mathrm{O} \mathrm{L}_{\text {fib }}$, único parâmetro anatômico em que o efeito do fator método foi significativo, apresentou distinção significativa na aplicação da ANOVA somente na comparação entre os lenhos juvenis das madeiras de açoita-cavalo $(\mathrm{F}=22,31 ; \mathrm{p}<0,01)$ e nogueira-pecã

Tabela 1 - Modelos matemáticos ajustados para a segregação entre os lenhos juvenil e adulto de açoita-cavalo, nogueira-pecã e plátano.

Table 1 - Mathematical models adjusted for segregation between juvenile and mature woods of açoita-cavalo, pecan and sycamore.

\begin{tabular}{|c|c|c|c|c|}
\hline \multirow[t]{2}{*}{ Equação } & & $\mathrm{R}^{2}$ & Syx & Valor F \\
\hline & Açoita-cavalo & & & \\
\hline a & $\mathrm{L}_{\mathrm{fib}}=1158,54+34,8631 \cdot \mathrm{A}_{\mathrm{cres}}$ & 0,62 & 190 & 6215 \\
\hline $\mathrm{b}$ & $\mathrm{L}_{\text {fib }}=1948,83-3,13954 \cdot \mathrm{A}_{\text {cres }}^{\text {cres }}$ & 0,03 & 205 & 140 \\
\hline \multirow[t]{2}{*}{ C } & $\mathrm{L}_{\mathrm{fib}}=1260,34+31,2316 \cdot \mathrm{A}_{\mathrm{cres}}^{\mathrm{cres}}-0,390719 \cdot \mathrm{A}_{\text {cres }}{ }^{2}$ & 0,38 & 218 & 2762 \\
\hline & Nogueira-pecã & & & \\
\hline a & $\mathrm{L}_{\mathrm{fib}}=1000,24+18,2771 \cdot \mathrm{A}_{\text {cres }}$ & 0,26 & 175 & 622 \\
\hline $\mathrm{b}$ & $L_{\text {fib }}=1371,06-4,33716 \cdot A_{c r e s}$ & 0,01 & 180 & 13 \\
\hline \multirow[t]{2}{*}{ C } & $\mathrm{L}_{\mathrm{fib}}^{\mathrm{fib}}=940,678+36,3516 \cdot \mathrm{A}_{\text {cres }}^{\text {cres }}-0,92304 \cdot \mathrm{A}_{\text {cres }}{ }^{2}$ & 0,25 & 173 & 440 \\
\hline & Plátano & & & \\
\hline a & $\mathrm{L}_{\mathrm{fib}}=1344,96+42,1538 \cdot \mathrm{A}_{\mathrm{cres}}$ & 0,33 & 244 & 612 \\
\hline $\mathrm{b}$ & $L_{\text {fib }}=2062,28-9,99406 \cdot A_{\text {cres }}^{\text {cres }}$ & 0,01 & 265 & 8 \\
\hline C & $\mathrm{L}_{\mathrm{fib}}=1246,67+77,0673 \cdot \mathrm{A}_{\text {cres }}^{\text {cres }}-2,2697 \cdot \mathrm{A}_{\mathrm{cres}}{ }^{2}$ & 0,32 & 248 & 501 \\
\hline
\end{tabular}

Em que: $\mathrm{L}_{\mathrm{fib}}=$ comprimento de fibra $(\mu \mathrm{m}) ; \mathrm{A}_{\text {cres }}=$ anel de crescimento; a = modelo de regressão linear da parte ascendente - método das retas de regressão; $b=$ modelo de regressão linear da parte constante - método das retas de regressão; $c=$ modelo polinomial - método do incremento anual; $\mathrm{R}^{2}$ = coeficiente de determinação; Syx = erro médio padrão ( $\mathrm{A}_{\text {cres }}$ ); e $\mathrm{F}=$ valor $\mathrm{F}$ calculado.

Where: $L_{\text {ib }}=$ fiber length $(\mathrm{um}) ; A_{\text {a }}=$ growth ring; $a$ = linear regression model of the ascending part - regression lines method; $b=$ linear regression model of the constant part-regression lines method; $c=$ polynomial model - annual increment method; $R^{2}=$ coefficient of determination; Syx = standard error $\left(A_{\text {cres }}\right)$; and $F=F$ value calculated.

Revista Árvore, Viçosa-MG, v.38, n.5, p.943-950, 2014 
$(\mathrm{F}=4,66 ; \mathrm{p}<0,05)$. A comparação dessa propriedade anatômica entre métodos só foi realizada nas madeiras de açoita-cavalo e nogueira-pecã, já que na madeira de plátano o anel de crescimento definido como o de início do lenho adulto foi o mesmo nas duas metodologias.
Nas Tabelas 2, 3, 4 e 5, apresentam-se as comparações múltiplas entre as propriedades anatômicas $\left(\mathrm{L}_{\text {fib }}, \varnothing_{\text {fib }}\right.$, $\varnothing_{\text {lume }}$ e $_{\text {fib }}$ ), em razão dos fatores analisados (Espécie, Tipo de lenho e Método), respeitando-se às interações que se mostraram significativas, segundo a ANOVA multifatorial.

Tabela 2 - Comparações múltiplas das médias do comprimento de fibra nas interações entre espécie e tipo de lenho. Table 2 -Multiple comparisons of the mean fiber length in the interactions among species and type of wood.



Letras iguais, minúsculas nas linhas e maiúsculas nas colunas não possuem diferença significativa de acordo com testes HSD de Tukey $(\mathrm{p}<0,01)$. Coeficiente de variação entre parênteses.

Same letters, lowercase in the lines and uppercase in the columns have no significant difference according to Tukey HSD tests $(p<0.01)$. Values in parentheses correspond to the coefficient of variation.

Tabela 3 - Comparações múltiplas das médias do diâmetro de fibra nas interações entre espécie e tipo de lenho. Table 3 -Multiple comparisons of the mean fiber diameter in the interactions among species and type of wood.

\begin{tabular}{|c|c|c|c|}
\hline & \multicolumn{3}{|c|}{ Diâmetro de fibra $(\mu \mathrm{m})$} \\
\hline & Açoita-cavalo & Nogueira-pecã & Plátano \\
\hline & \multicolumn{3}{|c|}{ Método das retas de regressão } \\
\hline Lenho Juvenil & $20,35_{(15,15)} \mathrm{Ab}$ & $17,04_{(13,77)}$ Аа & $23,79_{(17,66)}$ Ac \\
\hline \multirow[t]{2}{*}{ Lenho Adulto } & $21,55_{(14,87)}^{(11,15)} \mathrm{Bb}$ & $17,64_{(13,79)}^{(13,7)} \mathrm{Ba}$ & $25,42_{(16,20)}^{(1,60)} \mathrm{Bc}$ \\
\hline & \multicolumn{3}{|c|}{ Método do incremento anual } \\
\hline Lenho Juvenil & $20,22_{(14,92)} \mathrm{Ab}$ & $16,99_{(13,68)} \mathrm{Aa}$ & $23,79_{(17,66)}$ Ac \\
\hline Lenho Adulto & $21,55_{(14,92)}^{(1,4)} \mathrm{Bb}$ & $17,61_{(13,86)}^{(13,68)} \mathrm{Ba}$ & $25,42_{(16,20)}^{(1,60)} \mathrm{BC}$ \\
\hline
\end{tabular}

Letras iguais, minúsculas nas linhas e maiúsculas nas colunas não possuem diferença significativa de acordo com o teste HSD de Tukey $(\mathrm{p}<0,01)$. Coeficiente de variação entre parênteses.

Same letters, lowercase in the lines and uppercase in the columns have no significant difference according to Tukey HSD tests $(p<0.01)$. Values in parentheses correspond to the coefficient of variation.

Tabela 4 - Comparações múltiplas das médias do diâmetro de lume nas interações entre espécie e tipo de lenho. Table 4-Multiple comparisons of the mean lumen diameter in the interactions between species and type of wood.

\begin{tabular}{|c|c|c|c|}
\hline & \multicolumn{3}{|c|}{ Diâmetro de lume $(\mu \mathrm{m})$} \\
\hline & Açoita-cavalo & Nogueira-pecã & Plátano \\
\hline & \multicolumn{3}{|c|}{ Método das retas de regressão } \\
\hline Lenho Juvenil & $11,48_{(21,60)}$ Ac & $8,20_{(25,75)}$ AA & $9,01_{(33,19)} \mathrm{Bb}$ \\
\hline \multirow[t]{2}{*}{ Lenho Adulto } & $11,81_{(19,45)}^{(21,60)} \mathrm{Bc}$ & $8,24_{(27,18)}^{(25,75)} \mathrm{Ab}$ & $7,30_{(36,89)}^{(33,19)} \mathrm{Aa}$ \\
\hline & \multicolumn{3}{|c|}{ Método do incremento anual } \\
\hline Lenho Juvenil & $11,42_{(21,37)}$ Ac & $8,20_{(25,22)}$ AA & $9,01_{(33,19)} \mathrm{Bb}$ \\
\hline Lenho Adulto & $11,82_{(19,65)}^{(21,37)} \mathrm{BC}$ & $8,24_{(27,42)}^{(25,22)} \mathrm{Ab}$ & $7,30_{(36,89)}^{(33,19)} \mathrm{Aa}$ \\
\hline
\end{tabular}

Letras iguais, minúsculas nas linhas e maiúsculas nas colunas não possuem diferença significativa de acordo com o teste HSD de Tukey $(\mathrm{p}<0,01)$. Coeficiente de variação entre parênteses.

Same letters, lowercase in the lines and uppercase in the columns have no significant difference according to Tukey HSD tests $(p<0.01)$. Values in parentheses correspond to the coefficient of variation. 
Tabela 5 - Comparações múltiplas das médias da espessura de fibra nas interações entre espécie e tipo de lenho. Table 5-Multiple comparisons of the mean fiber thickness in the interactions between species and type of wood.

\begin{tabular}{|c|c|c|c|}
\hline & \multicolumn{3}{|c|}{ Espessura de fibra $(\mu \mathrm{m})$} \\
\hline & Açoita-cavalo & Nogueira-pecã & Plátano \\
\hline & \multicolumn{3}{|c|}{ Método das retas de regressão } \\
\hline Lenho Juvenil & $4,43_{(21,94)} \mathrm{Aa}$ & $4,41_{(21,94)} \mathrm{Aa}$ & $7,39_{(22,92)} \mathrm{Ab}$ \\
\hline \multirow[t]{2}{*}{ Lenho Adulto } & $4,87_{(20,36)} \mathrm{Bb}$ & $4,69_{(19,77)} \mathrm{Ba}$ & $9,06_{(8,98)} \mathrm{BC}$ \\
\hline & \multicolumn{3}{|c|}{ Método do incremento anual } \\
\hline Lenho Juvenil & $4,39_{(20,08)} \mathrm{Aa}$ & $4,39_{(21,91)} \mathrm{Aa}$ & $7,39_{(22.92)} \mathrm{Ab}$ \\
\hline Lenho Adulto & $4,86_{(20,33)} \mathrm{Bb}$ & $4,68_{(19,99)} \mathrm{Ba}$ & $9,06_{(8,98)} \mathrm{BC}$ \\
\hline
\end{tabular}

Letras iguais, minúsculas nas linhas e maiúsculas nas colunas não possuem diferença significativa de acordo com o teste HSD de Tukey $(\mathrm{p}<0,01)$. Coeficiente de variação entre parênteses.

Same letters, lowercase in the lines and uppercase in the columns have no significant difference according to Tukey HSD tests $(p<0.01)$. Values in parentheses correspond to the coefficient of variation.

\section{DISCUSSÃO}

Os resultados verificados neste estudo corroboram os descritos por outros autores, de modo a possibilitar a segregação dos lenhos juvenil e adulto mediante a observação da distribuição dos dados no gráfico $\mathrm{A}_{\text {cres }}$ versus $\mathrm{L}_{\text {fib }}$. Isso tanto com base no método das retas de regressão (LOO et al., 1985; KRAHMER, 1986; GATTO et al., 2013; PALERMO et al., 2013) quanto no método do incremento anual (SHIOKURA, 1982; OLUWAFEMI, 2007; NUGHORO et al., 2012).

O efeito significativo dentro do fator "Método" verificado para o $\mathrm{L}_{\text {fib }}$ indica que realmente esse parâmetro é o melhor entre os estudados nesta pesquisa para determinação das zonas contidas pelos lenhos juvenil e adulto. Resultado concordante com outros estudos antecedentes (BENDTSEN, 1978; KRAHMER, 1986).

Em relação ao $\mathrm{L}_{\text {fib }}$, os coeficientes de variação foram menores no lenho adulto, inferindo maior homogeneidade em sua formação (Tabela 2). Os maiores coeficientes de variação verificados no lenho juvenil, em comparação com o lenho adulto, nos valores médios de $\mathrm{L}_{\text {fib }}$ ocorrem, pois a taxa de mudança em propriedades anatômicas, como o comprimento de fibra, é maior no lenho juvenil, o qual, a partir da medula, vai assumindo gradativamente os valores médios encontrados no lenho adulto (BENDTSEN, 1978; KRAHMER, 1986).

Além de $\mathrm{L}_{\text {fib }}$, para $\varnothing_{\text {fib }}$ e $\mathrm{e}_{\mathrm{fib}}$, as Tabelas 2, 3, 4 e 5 indicaram que, de modo geral, em comparação com o lenho juvenil, o lenho adulto apresentou os maiores valores das propriedades anatômicas avaliadas. Assim como neste estudo, outros autores também evidenciaram valores mais elevados para as propriedades anatômicas $\left(L_{\text {fib }}, \varnothing_{\text {fib }}, \varnothing_{\text {lume }}\right.$ e e $e_{\text {fib }}$ ) no lenho adulto em relação ao lenho juvenil (BENDTSEN, 1978; SENFT et al., 1985; YEH et al., 2006; NUGHORO et al., 2012; GATTO et al., 2013).

Esse fenômeno ocorre devido ao processo de amadurecimento do câmbio, o qual se dá gradativamente em razão do mecanismo de diferenciação e especialização das células meristemáticas. Assim, a maturação das características biométricas das células estruturais não ocorre ao mesmo tempo, pois cada novo tecido formado não necessariamente tem a mesma finalidade da respectiva célula meristemática precursora (EVERT, 2006).

Na comparação entre comprimento de fibra e as outras propriedades anatômicas da madeira analisadas neste estudo, no sentido medula-casca, o comportamento não ocorre de forma semelhante, pois o comprimento de fibra estabiliza-se antes das demais propriedades (ZOBEL; BUIJTENEN, 1989), o que explica a discrepância verificada neste estudo para o $\mathrm{L}_{\text {fib }}$ em comparação com $\varnothing_{\text {fib }}, \varnothing_{\text {lume }} \mathrm{e}_{\text {fib }}$. Portanto, essa característica pode ser citada como fator que dificulta a demarcação das zonas juvenil e adulta de diferentes madeiras.

O $\varnothing_{\text {lume }}$ (Tabela 4) denotou comportamento diferenciado em relação ao fator espécie, pois nas madeiras de açoita-cavalo e nogueira-pecã esse parâmetro anatômico apresentou maiores valores para o lenho adulto, comparado ao lenho juvenil, diferença essa que foi estatisticamente significativa apenas para a primeira espécie. Com relação à madeira de plátano, verificou-se resultado contrário, em que o $\emptyset_{\text {lume }}$ foi maior no lenho juvenil. As diferenças nas magnitudes dos parâmetros anatômicos entre espécies podem ser justificadas em razão das diferenças de idade, mas, 
principalmente, pelas diferenças intrínsecas que existem entre os indivíduos arbóreos das três madeiras analisadas.

Conforme Burger e Richter (1991), o $\varnothing_{\text {lume }}$ do lenho juvenil é maior, pois suas células são frequentemente respectivas ao lenho inicial, e estas têm por função a condução na planta. No entanto, neste estudo somente o lenho inicial de cada anel foi selecionado para a maceração; dessa forma, esse resultado só pode ser atribuído a aspectos intrínsecos a cada madeira.

Apesar de não terem sido verificadas diferenças expressivas nas idades de segregação dos lenhos obtidas pelos dois métodos avaliados, deve-se enfatizar que a metodologia do incremento anual pode ser considerada mais confiável, pois seu procedimento não leva em conta nenhum tipo de avaliação decisória do experimentador. No caso do método das retas de regressão, as regiões do gráfico que dão origem às retas são escolhidas visualmente, o que implica fonte de erro experimental que pode ser considerada como erro aleatório, pois se trata de parcela imprevisível da probabilidade de falha no experimento.

\section{CONCLUSÕES}

Os métodos de retas de regressão e do incremento anual indicaram que a madeira de açoita-cavalo segregou aos 21 e 19 anos e a madeira de nogueira-pecã, aos 16 e 14 anos, respectivamente. A madeira de plátano segregou aos 14 anos para as duas metodologias. Com base nos valores médios de propriedades anatômicas das fibras incluindo comprimento, diâmetro total e diâmetro do lume, as metodologias utilizadas estimaram idades de delimitação entre os lenhos juvenil e adulto semelhantes, exceto para o comprimento de fibra do lenho juvenil de açoita-cavalo e nogueira-pecã. O lenho adulto apresentou maiores níveis dessas características da fibra do que o lenho juvenil, exceto para o diâmetro de lume da madeira de plátano. A metodologia do incremento anual pode ser tida como preferencial razão da fonte de erro experimental, verificada no método das retas de regressão.

\section{REFERÊNCIAS}

ALTEYRAC, J. et al. Influence of stand density on ring width and wood density at different sampling heights in black spruce (Picea mariana (Mill.) B.S.P.). Wood Fiber Science, v.37, n.1, p.83-94, 2005.
ALTEYRAC, J.; CLOUTIER, A.; ZHANG, S. Y. Characterization of juvenile wood to mature wood transition age in black spruce (Picea mariana (Mill.) B.S.P.) at different stand densities and sampling heights. Wood Science and Technology, v.40, n.2, p.124-138, 2006.

BAL, B. C. Some physical and mechanical properties of thermally modified juvenile and mature black pine wood. European Journal of Wood and Wood Products. v.72, n.1, p.61-66, 2014.

BAO, F. C. et al. Differences in wood properties between juvenile wood and mature wood in 10 species grown in China. Wood Science and Technology, v.35, n.4, p.363-375, 2001.

BENDTSEN, B. A. Properties of wood from improved and intensively managed trees. Forest Products Journal, v.28, n.10, p.61-71, 1978.

BOUSLIMI, B.; KOUBAA, A.; BERGERON, Y. Effects of biodegradation by brown-rot decay on selected wood properties in eastern white cedar (Thuja occidentalis L.). International Biodeterioration \& Biodegradation. v.87, n.2, p.87-98, 2014.

BURGer, L. M.; RICHTER, H. G. Anatomia da madeira. São Paulo: Nobel, 1991. 154p.

DELUCIS, R. A. et al. Segregação e variação radial das propriedades físicas da madeira juvenil e adulta de cedro (Cedrela fissilis Vellozo).

Scientia Florestalis, v.100, n.4, p.549-556, 2013.

DÜNISCH, O.; RICHTER, H. G.; KOCH, G. Wood properties of juvenile and mature heartwood in Robinia pseudoacacia L. Wood Science and Technology, v.44, n.2, p.301-313, 2010.

EMPRESA BRASILEIRA DE PESQUISA AGROPECUÁRIA - EMBRAPA. Centro Nacional de Pesquisa de Solos. Sistema brasileiro de classificação de solos. Brasília: 1999. 412p.

EVERT, R. F. Esau's plant anatomy: meristems, cells, and tissues of the plant body: their structure, function, and development. 3.ed. New Jersey: John Wiley \& Sons, 2006. 601p.

Revista Árvore, Viçosa-MG, v.38, n.5, p.943-950, 2014 
GATTO, D. A. et al. Proportion of juvenile wood of açoita-cavalo, pecan and London plane wood. International Wood Products Journal, v.4, n.1, p.33-36, 2012.

KRAHMER, R. Fundamental anatomy of juvenile and mature wood. In: A TECHNICAL

WORKSHOP: juvenile wood what does it mean to forest management and forest products, 1985, Washington. Proceedings... Madison: Forest Products Research Society, 1986. p.12-16.

LARA PALMA, H. A.; LEONELLO, E. C.;

BALLARIN, A. W. Demarcação da madeira juvenil e adulta de Corymbia citriodora. Cerne, v.16, p.141-148, 2010. (Suplemento)

LOO, J. A.; TAUER, C. G.; MCNEW, R. W. Genetic variation in the time of transition from juvenile to mature wood in loblolly pine (Pinus taeda L.).

Silvae Genetetica, v.34, n.1, p.14-19, 1985.

LOURENÇON, T. V. et al. Determinação da idade de transição entre lenho juvenil e adulto de três espécies florestais por meio de suas propriedades mecânicas. Floresta e Ambiente, v.21, n.2, p.251-260, 2014.

NUGHORO, W. D. et al. Radial variations in the anatomical characteristics and density of the wood of Acacia mangium of five different provenances in Indonesia. Journal of Wood Science, v.58, n.3, p.185-194, 2012.

OLUWAFEMI, O. A. Wood properties and selection for rotation length in Caribbean Pine (Pinus caribaea Morelet) grown in Afaka, Nigeria.

\author{
American- Eurasian Journal \\ Agriculture \& Enviromental Science, \\ v.2, n.4, p.359-363, 2007.
}

PALERMO, G. P. M. et al. Delimitação entre os lenhos juvenil e adulto de Pinus elliottii Engelm. Árvore, v.37, n.1, p.191-200, 2013.

SAMUELSON, L. J. et al. Relationships between climate, radial growth and wood properties of mature loblolly pine in Hawaii and a northern and southern site in the southeastern United States. Forest Ecology and Management, v.310, n.15, p.786-795, 2013.

SENFT, J. F.; BENDTSEN, B. A.; GALLIGAN, W. L. Weak wood, fast-grown trees make problem lumber. Journal of Forestry, v.83, n.8, p.476$484,1985$.

SHIOKURA, T. Extent and differentiation of the juvenile wood zone in coniferous tree trunks. Wood Fiber Science, v.28, n.2, p.85-90, 1982.

YEH, T. et al. Morfological and chemical variations between juvenile wood, mature wood and compression wood of loblolly pine (Pinus taeda L.). Holzforschung, v.60, n.1, p.1-8, 2006.

ZHU, J.; NAKANO, T.; HIRAKAWA, Y. Effects of radial growth rate on selected indices for juvenile and mature wood of the Japanese larch. Journal of Wood Science, v.46, n.6, p.417-422, 2000.

ZOBEL, B. J.; van BUIJTENEN, J. P. Wood variation: its causes and control. New York: Springer-Verlag, 1989. 363p. 